\section{Inflammasome jigsaw puzzles}

Inflammasomes are multiprotein complexes that assemble in response to infection or tissue damage to activate caspase- 1 and induce the secretion of interleukin $1 \beta$ (IL-1 $\beta$ ) and IL-18. In Science, Shenoy et al. show that GBP5 is specifically required for assembly of the NLRP3 inflammasome in human and mouse macrophages in response to live bacteria and soluble agents but not in response to crystalline agents. GBP5 is a member of a family of guanylate-binding proteins with recently identified immunological functions, and its expression is optimally induced by stimulation with lipopolysaccharide plus interferon- $\gamma$. GBP5 directly interacts with NLRP3 and undergoes tetramerization in a GTPase-independent manner. The GBP5 tetramer promotes oligomerization of the adaptor ASC via its interaction with NLRP3, which suggests that this newly identified inflammasome component can coordinate the modular assembly requirements of the inflammasome.

Science 27, 481-485 (2012)

\section{Pathogen-tailored $\mathrm{T}_{H} 17$ cells}

Patients with genetic defects in cells of the $\mathrm{T}_{\mathrm{H}} 17$ subset of helper $\mathrm{T}$ cells suffer recurrent infections with Candida albicans and Staphylococcus aureus. In Nature, Zielinski et al. use whole-microbe pulsing of monocytes to stimulate naive and memory human $\mathrm{CD} 4^{+} \mathrm{T}$ cells to assess the polarizing conditions and effector characteristics induced by complex microbes. C. albicans-specific $\mathrm{T}_{\mathrm{H}} 17$ cells mainly produce both IL-17 and interferon- $\gamma\left(\right.$ IFN- $\gamma$ ), whereas $S$. aureus-specific $\mathrm{T}_{\mathrm{H}} 17$ cells mostly produce only IL-17 but can induce IL-10 after restimulation. IL-6, IL-23 and IL- $1 \beta$ contribute to the $\mathrm{T}_{\mathrm{H}} 17$ differentiation induced by each pathogen, but IL-1 $\beta$ is essential in C. albicans-induced $\mathrm{T}_{\mathrm{H}} 17$ differentiation. IL-1 $\beta$ is needed to counteract the inhibitory effect of IL-12, which is detected only in C. albicans cultures, to induce cells that produce both IL-17 and IFN- $\gamma$ and to inhibit IL-10 production. IL-1 $\beta$ has similar effects on memory T cells, which suggests that IL- $1 \beta$ primes cells not only in lymphoid organs but also in target tissues.

Nature (1 April 2012) doi:10.1038/nature10957

\section{Building blocks of hematopoiesis}

Specialized tissue microenvironments direct and maintain the development of hematopoietic stem cells, but the cellular and molecular interactions of this process are still being determined. In Cell, Calderon and Boehm use a modular approach to delineate the rules of hematopoiesis. Through reconstitution of the thymic epithelium of nude mice that lack hematopoietic function with various combinations of chemokines (CCL25 and CXCL12), the cytokine SCF and the Notch ligand DLL4, the thymic epithelium is made functional to support different progenitor populations. For example, CXCL12, DLL4 and SCF together support T cell progenitors, whereas CCL25, CXCL12 and SCF are biased toward B cells. In total, the authors generate 15 different combinations of factors, each with its own functionality. This study identifies the surprisingly minimalist rules that can govern hematopoiesis. ZF Cell 149, 159-172 (2012)

Written by Laurie A. Dempsey, Zoltan Fehervari \& Ioana Visan

\section{TSLP underlies the atopic march}

Atopic dermatitis is a common allergic skin disorder that often precedes the development of allergy in distal tissues such as the lung. In Mucosal Immunology, Ziegler and colleagues describe how the epithelial cell-associated cytokine TSLP can drive this so-called 'atopic march' from one tissue to another. Intradermal injection of mice with TSLP plus antigen results in asthma after subsequent lung challenge with that same antigen alone. TSLP expression in the lung is not required for the manifestation of asthma, but an antigen-specific memory $\mathrm{CD} 4^{+} \mathrm{T}$ cell response is essential for this. Similar results are obtained after inducible expression of TSLP by keratinocytes. IL-25 is another epithelial cell-derived cytokine linked to allergy; however, it has no role in the dissemination of allergy from the skin to the lungs. Although mice can be made tolerant to asthma orally, this is possible only if done before skin sensitization, a finding with implications for the translation of such immunotherapy to patients.

Mucosal Immunol. 5, 342-351 (2012)

\section{TCR tuning}

The tuning of T cell antigen receptor (TCR) responsiveness allows immature thymocytes to be positively selected by complexes of self peptide and major histocompatibility complex molecules of lower affinity but prevents mature $T$ cells from responding to similar complexes in the periphery. In the Proceedings of the National Academy of Science, Laufer and colleagues show the kinase Lck redistributes in 'tuned' thymocytes to increase the threshold required for the activation of mature T cells. Tuning occurs in a major histocompatibility complex-dependent manner after positive selection to downregulate TCR sensitivity. In 'untuned' or preselection $\mathrm{CD} 4^{+} \mathrm{CD} 8^{+}$double-positive thymocytes, more phosphorylated Lck is associated with the TCR CD3 $\zeta$ chain. In contrast, more Lck associates with CD4 in 'tuned' thymocytes, and these complexes localize in membrane-associated protein islands separate from $\mathrm{CD} 3 \zeta$. This separation of protein islands in 'tuned' cells increases the threshold for TCR activation and thus requires agonist peptides of higher affinity, which thereby lessens the potential for $\mathrm{T}$ cell autoreactivity.

$L A D$

Proc. Natl. Acad. Sci. USA (23 April 2012) doi:10.1073/ pnas.1119272109

\section{Good microbes}

Increasing evidence supports the proposal of a role for commensal microorganisms in shaping systemic immune responses. In Nature Medicine, Artis and colleagues show that commensal bacteria can suppress the switching of B cells to immunoglobulin E (IgE). Germ-free mice and mice treated with broad-spectrum antibiotics have higher concentrations of circulating IgE. In turn, higher IgE concentrations correlate with more basophils and T helper type 2 cells and skewing toward type 2 immune responses in these mice. IgE induces upregulation of the receptor for IL-3 (CD123) in bone marrow precursor cells, which results in more basophil progenitor cells. Inhibition of IgE production or blockade of the receptor FceRI $\alpha$ diminishes the basophilia of the antibiotic-treated mice. Intriguingly, B cell-intrinsic expression of the adaptor MyD88 suppresses IgE production, basophilia and enhanced susceptibility to atopic disease. These findings may explain the greater incidence of atopic diseases as a result of more antibiotic use. LAD Nat. Med. 18, 538-546 (2012) 